\title{
Indicadores altmétricos em periódicos brasileiros da Ciência da Informação do Brasil
}

\author{
Ilídio Lobato Ernesto Manhique \\ Escola Superior de Jornalismo de Moçambique, Maputo, Moçambique \\ ilidiolobato@gmail.com
}

Fernando de Assis Rodrigues

Universidade Estadual Paulista Júlio Mesquita Filho - UNESP, Programa de Pós-Graduação em Ciência da Informação, Marilia, SP, Brasil

fernando@rodrigues.pro.br

Ricardo César Gonçalves Sant'Ana

Universidade Estadual Paulista Júlio Mesquita Filho - UNESP, Programa de Pós-Graduação em Ciência da Informação, Marilia, SP, Brasil

ricardosantana@marilia.unesp.br

Helen de Castro Silva Casarin

Universidade Estadual Paulista Júlio Mesquita Filho - UNESP, Programa de Pós-Graduação em Ciência da Informação, Marilia, SP, Brasil

helen.casarin@gmail.com

DOI: https://doi.org/10.26512/rici.v12.n2.2019.9156

Recebido/Recibido/Received: 2018-06-14

Aceitado/Aceptado/Accepted: 2019-01-28

Resumo: 0 crescente volume informacional impulsionado pelo desenvolvimento das Tecnologias de Informação e Comunicação ampliou o foco sobre a busca por visibilidade de publicações no campo científico, elevando o grau de relevância de questões como a do reconhecimento acadêmico de pesquisadores, resultando no questionamento de medidas tradicionais de avaliação de impacto acadêmico. Este estudo tem como objetivo identificar a visibilidade de parte da produção científica brasileira da Ciência da Informação, a partir dos periódicos indexados na Base de Dados Referenciais de Artigos de Periódicos em Ciência da Informação (BRAPCl), com o uso de dados de indicadores altmétricos de serviços de Redes Sociais Online e de gerenciamento de referências coletados via Altmetric Bookmarklet. Constatou-se que os dados altmétricos ainda possuem baixa aderência no contexto dos periódicos brasileiros relacionados com a Ciência da Informação. Entre os fatores envolvidos destaca-se a inexistência de identificadores em aproximadamente oitenta por cento dos periódicos indexados pela BRAPCl e a baixa identificação de menções às publicações nos serviços de Redes Sociais Online. Conclui-se que estas métricas alternativas para complementar as métricas tradicionais ainda necessitam de aprofundamento teórico e destaca-se, como requisito, a necessidade de instrumentalização técnica por parte dos editores.

Palavras-chave: Altmetria. Redes Sociais. Ciência da Informação. BRAPCI. Brasil. 
Resumen: El creciente volumen informacional impulsado por el desarrollo de las Tecnologías de Información y Comunicación amplió el foco sobre la búsqueda de visibilidad de publicaciones en el campo científico, elevando el grado de relevancia de aspectos como do reconocimiento académico de investigadores, resultando en el cuestionamiento de las medidas tradicionales de evaluación de impacto académico. Lo objecto de este estudio és identificar la visibilidad de parte de la producción científica brasileña de Ciencia de la Información, a partir de las revistas científicas clasificado en la BRAPCI (Base de Datos Referenciales de Artículos de Revistas de la Ciencia de la Información), con el uso de datos de indicadores altmétricos de servicios de redes sociales en línea y bases de datos vinculadas a sitios web de gestión de referencias. Se constató que los datos altmétricos aún tienen baja adherencia en el contexto de las revistas científicas brasileñas relacionadas con la Ciencia de la Información. Entre los factores envueltos se destaca la inexistencia de identificadores en aproximadamente el ochenta por ciento de las revistas científicas indexadas por la BRAPCl y la baja identificación de menciones en publicaciones en los servicios de redes sociales en línea. Se concluye que estas métricas alternativas para complementar las tradicionales necesitan de profundización teórica y se destaca, como requisito, la necesidad de instrumentalización técnica por parte de los editores.

Palabras clave: Altmetrics. Redes Sociales. Ciencia de la Información. Base de Datos Referenciales de Artículos de Revistas de la Ciencia de la Información. Brasil.

\section{Altmetrics indicators in Brazilian Journals of Information Science}

Abstract: The growing informational volume boosted by the development of Information and Communication Technologies has increased the focus on the seek for visibility of publications in the scientific field, raising the degree of relevance of the academic recognition by researchers, resulting in questions about the perception of the traditional measures of evaluation of the academic impact. The goal of this study is to identify the visibility of the Brazilian scientific production of Information Science area, from the periodicals indexed in BRAPCI (Referral Database of Journals Papers in Information Science), with the use of data of altmetric indicators from Online Social Networking services and databases related with reference management websites. The results show that the altmetric data still have a low level of adherence in the Brazilian journals context related to Information Science. Among the factors involved, stand out the lack of identifiers in approximately eighty percent of the journals indexed by BRAPCl and the low level of mentions on publications into the Online Social Networks services. It is concluded that these alternative metrics to complement the traditional metrics still need to be more explored theoretically and, as a requirement, technically through an implement process by publishers.

Keywords: Altmetrics. Social Networks. Information Science. Database of references from Information Science Journals. Brazil.

\section{Introdução}

O campo científico exerce a função primária de produção e socialização do conhecimento que é discutido, reutilizado, questionado e/ou apropriado pela comunidade dentro e fora do ambiente acadêmico. A partir da segunda metade do século passado, com o crescimento do volume informacional resultante do desenvolvimento das Tecnologias de Informação e Comunicação (TIC), bem como pela crescente especialização da ciência, o campo científico converteu-se em centro de competitividade, no qual a busca pelo reconhecimento acadêmico dos autores tornou-se num dos seus aspectos dominantes (SARACEVIC, 1996; SMALL, 2004).

Estas questões, associadas às melhorias em produtividade, suscitaram indagações relativas à qualidade das publicações científicas, o que originou a criação de mecanismos de filtragem das publicações acadêmicas dentro das comunidades científicas. Para Meadows, “[...] 
a forma de avaliar a qualidade consiste em avaliar o nível de interesse dos outros pela pesquisa. O método mais simples de medir isso é por meio de citações dessa pesquisa na bibliografia ulterior" (MEADOWS, 1999, p. 89)

As citações são parte importante do rol de parâmetros de qualidade e é um recurso amplamente aceito no âmbito acadêmico, especialmente por editores e agências de fomento. No entanto, o uso das citações como indicadores para mensurar a qualidade de publicações científicas, bem como para aferir a autoridade de determinados autores, requer uma redobrada atenção, porque entre as diversas razões que levam os pesquisadores a citar uma determinada publicação nem todas estão relacionadas com a influência intelectual. Dentre os fatores que influenciam a citação destacam-se os psicológicos, sociais, o prestígio do autor citado, o idioma de publicação citada, o prestígio do periódico entre os outros (MEADOWS, 1999; SPINAK, 1998; MACIAS-CHAPULA, 1998).

Outro fator de complexidade no processo de avaliação da qualidade está relacionado a dificuldade avaliar publicações de diferentes idiomas, especialmente pelo prestígio já estabelecido às publicações que estejam vinculadas às bases de dados de indexação de periódicos científicos de países europeus e dos Estados Unidos da América. Em geral, uma das regras para o aceite da publicação nestas bases de dados é a predominância da publicação na língua inglesa (MACIA-CHAPULA, 1998; BORREGO, 2014), o que contribui para a "exclusão cognitiva" (KURAMOTO, 2007).

Associados a esta problemática, o desenvolvimento das TIC permitiu o surgimento de serviços de Redes Sociais Online (RSO), propiciando interfaces para a interlocução dos indivíduos participantes (RODRIGUES, 2017; RODRIGUES; SANT'ANA, 2016). Estas transformações relacionadas ao uso de TIC nas atividades profissionais aprofundaram a emergência de um movimento de reformas e de novas propostas de avaliação do impacto da atividade científica ao novo contexto, sobretudo, pelos serviços de RSO.

Dentre estas movimentações, surgiram propostas de utilização de métricas alternativas, também denominada como altmetrias ou altmétricas, com origem no termo na língua inglesa altmetrics. As altmetrias são aderentes a esta necessidade de incluir outros elementos que demonstrem a atenção e a apropriação da produção científica dentro e fora do contexto acadêmico. Esta proposta teve origens no manifesto de Priem et al. (2010), como uma forma de contraponto as avaliações de impacto do Journal of Impact Factor, que tem como base a análise por citações como critério exclusivo de qualidade.

Apesar de ser um fenômeno recente, existem diversas definições relacionadas a altmetria. Borrego (2014) propõe uma compilação por relevância, descrevendo-as como parte de uma categoria de indicadores que medem o impacto da investigação quantificando a sua 
presença nas páginas web com caráter social e bibliográfico, no que respeita ao número de tuítes, menções em blogs, inclusão em marcadores sociais e presença em gestores bibliográficos. Estes indicadores têm como diferencial o fato de quantificarem o impacto social das publicações científicas por meio das redes sociais e de outras plataformas de interação online disponibilizadas pelas Web 2.0 (BORNMANN, 2014; GARCIA et al., 2014).

Segundo Borrego (2014, p. 353), as altmetrias fornecem uma visão complementar às citações no processo de avaliação da ciência e "[...] podem ser úteis como novos metadados que ajudam o leitor na busca de informação e ao bibliotecário na identificação dos hábitos e necessidades de informação de seus usuários".

$\mathrm{Na}$ construção dos indicadores de altmetria, os processos de coleta, de armazenamento e de visualização de dados são imprescindíveis, pois este tipo de análise requer dados armazenados em formatos distintos e em diversos recursos, tais como: de bases de dados, de serviços de RSO e de Sistemas Gerenciadores de Conteúdo. São estes dados que permitirão a avaliação do impacto acadêmico e social de uma determinada publicação (PRIEM et al., 2010). É a partir da coleta de dados de acesso e de registros de atividades que é possível mapear a atenção que uma publicação conquista no seio de uma determinada comunidade.

$\mathrm{Na}$ literatura, estes são descritos como alternativos às métricas de avaliação tradicionais, porém com abordagem distinta, principalmente com relação a instantaneidade dos dados compartilhados em redes sociais online e de ferramentas de gerenciamento de referências e os formatos de dados estruturados para compartilhamento de referências ALPERIN, 2013). A aplicação adequada destas métricas pode contribuir para visualizar o impacto da produção acadêmica de países, historicamente, excluídos pelas métricas tradicionais de impacto. Nesse sentido, as métricas alternativas pressupõem um processo de democratização da atribuição da recompensa acadêmica (HAUSTEIN, 2016), visto que as RSO são espaços abertos de interlocução entre diversos agentes sociais.

Como fator delimitador, a coleta de dados para altmetria é dependente do uso de identificadores para publicação científica, tais como: o DOI (Digital Object Identifier) e o PMID (PubMed Record ID). Apesar desta dependência, a relevância do uso destes indicadores para avaliação do impacto social das publicações tem se popularizado entre acadêmicos (ALPERIN, 2013; BORNMANN, 2014; GARCIA et. al, 2014; HAUSTEIN, 2016; BORREGO, 2014), que ressaltam a validade destas medidas em um contexto de mudança de padrões da comunicação e da apropriação científicas.

No Brasil, as pesquisas relacionadas com este objeto ainda estão na fase embrionária, e prevalece a dificuldade de se fazer análises por meio das altmétricas, pois existem poucos periódicos que publicam artigos com tais identificadores que permitem o cruzamento de dados 
(ARAÚJO, 2014). Existem alguns estudos que buscam aprofundar os seus pressupostos teóricos (VANTI; SANZ-CASADO, 2016) e outros que mapeiam a presença de dados altmétricos em determinados periódicos, sobretudo, os de Qualis A1 (ARAÚJO, 2015).

Não há evidência de existência de pesquisas que estudem a presença destas métricas em um número significativo de periódicos nacionais da $\mathrm{Cl}$. Este estudo tem como objetivo verificar a visibilidade de parte da produção científica brasileira da $\mathrm{Cl}$, a partir dos periódicos indexados na Base de Dados Referenciais de Artigos de Periódicos em Ciência da Informação (BRAPCI), com o uso de dados de indicadores alternativos (altmétricos) de serviços de Redes Sociais Online e bases de dados vinculadas a websites de gerenciamento de referências.

Como objetivos específicos, esta identificação se relaciona com os seguintes procedimentos: (i) verificar a aderência dos de dados altmétricos nos artigos de periódicos vinculados à $\mathrm{BRAPCl}$, (ii) selecionar periódicos científicos brasileiros que possuem elementos necessários a análise altmétrica na $\mathrm{BRAPCl}$, (iii) a partir dos resultados da análise altmétrica dos periódicos científicos selecionados, identificar aspectos demográficos sobre indivíduos que acessaram os artigos científicos e (iv) descrever quais são os tipos de dados altmétricos disponíveis nos periódicos.

Foram coletados dados sobre as publicações científicas dos periódicos da $\mathrm{Cl}$ indexados na BRAPCI, nos serviços de RSO Facebook e Twitter, em postagens em blogs e na ferramenta de gerenciamento de referências Mendeley. Foram descartados dados do indicador do serviço de rede social online Google+ e de websites de notícias, pois não foram verificadas menções nestas fontes.

O texto está estruturado em quatro seções: a introdução, com a contextualização do trabalho, definição do problema e dos objetivos, incluindo a apresentação dos aspectos conceituais ligados às altmetrias; a segunda seção, discorre sobre os procedimentos metodológicos adotados, com destaque para a caracterização da amostra e da ferramenta utilizada para a coleta de dados, e; a terceira seção, contendo a discussão e a análise dos dados e, por fim, as considerações finais.

\section{Percurso metodológico}

Este estudo é natureza exploratória e de caráter qualitativo e quantitativo, cujo objetivo foi verificar a visibilidade da produção científica da $\mathrm{Cl}$ brasileira nas mídias sociais e em outras plataformas online. Para McCormick et al. (2015), a coleta de dados em ambientes digitais dá a possibilidade de se observar o comportamento e a interação humana em tempo real e em escala global. Estes ambientes fornecem uma grande quantidade de dados disponibilizados pelos usuários sem que sejam solicitados pelo pesquisador. Dada a 
complexidade inerente ao espaço online, exige-se um conhecimento interdisciplinar, bem como a escolha de ferramentas adequadas para a coleta de dados, seja nas mídias sociais ou em quaisquer plataformas online.

A primeira etapa da pesquisa teve como base a pesquisa bibliográfica, que visava a construção de um referencial para o embasamento da pesquisa. Por meio deste procedimento, foram discutidos os aspectos conceituais, teóricos e históricos relacionados às altmétricas, incluindo as vantagens e algumas limitações técnicas para aplicação deste tipo de métricas para a avaliação do impacto social das publicações científicas.

A segunda etapa teve como foco a delimitação da amostra da pesquisa, abarcando o processo de seleção de periódicos para a constituição do corpus deste estudo. Os periódicos foram selecionados na $\mathrm{BRAPCl}$, que é uma base de dados que agrega a maioria das revistas científicas da $\mathrm{Cl}$ brasileira. Esta fase da pesquisa teve como objetivo identificar os periódicos brasileiros indexados nessa base de dados que tivessem o DOI, considerando que os dados altmétricos só podem ser obtidos se as publicações desses periódicos tiverem esse identificador.

No momento da coleta (Outubro e Novembro de 2017), a BRAPCl agregava 57 periódicos brasileiros da $\mathrm{Cl}$, ressalvando o fato de algumas revistas científicas terem sido descontinuadas e/ou com dados não atualizados. O levantamento para a identificação dos periódicos com o DOI foi feito diretamente em cada um dos 57 periódicos vinculados à BRAPCI. Desse levantamento foram identificados, apenas, 12 revistas da $\mathrm{Cl}$ que continham esse identificador - objetos de análise altmétrica (Quadro 1).

Importa frisar que não basta que o periódico tenha o DOI para que se consiga coletar os dados altmétricos dos seus artigos. É, também, necessário que cada artigo neles publicado possua esse identificador para uma coleta individualizada de dados altmétricos de cada publicação. No que concerne ao universo de periódicos vinculados à $\mathrm{BRAPCl}$, foi constatado que alguns periódicos (ex: Transinformação e Informação \& Sociedade), mesmo contendo esse identificador, não atribuíam o DOI aos seus artigos publicados e, por via disso, foram excluídos do corpus da pesquisa.

Não foram desconsiderados dados altmétricos provenientes de interfaces de bases de conhecimento que, em certos casos, adicionam identificadores e metadados extras em representações de artigos científicos dos periódicos indexados. Bases de conhecimento e repositórios institucionais têm adotado a prática de vincular identificadores às representações dos artigos indexados. Entretanto, esta prática não é garantia de disponibilidade destes identificadores via acesso direto pela interface dos periódicos e da adoção deste identificador por parte do periódico, o que permite cenários tais como: a existência de DOI para as 
representações de seus artigos científicos mas sem a existência do mesmo no periódico; o uso de DOI com valores diferentes para identificar as representações e a produção científica.

Quadro 1 - Dados dos periódicos científicos analisados

\begin{tabular}{|c|c|c|}
\hline Nome do periódico & ISSN & Endereço para acesso \\
\hline $\begin{array}{l}\text { A to } z \text { : novas práticas de informação } \\
\text { e conhecimento }\end{array}$ & $2237-826 X$ & https://revistas.ufpr.br/atoz \\
\hline Biblioteca Escolar em Revista & $2238-5894$ & http://revistas.ffclrp.usp.br/berev \\
\hline $\begin{array}{l}\text { Brazilian Journal of Information } \\
\text { Science: research trends }\end{array}$ & $1981-1640$ & $\begin{array}{l}\text { http://www2.marilia.unesp.br/revistas/index.php/bjis/ind } \\
\text { ex }\end{array}$ \\
\hline Ciência da Informação & 0100-1965 & http://revista.ibict.br/ciinf \\
\hline Comunicação \& Informação & $1415-5842$ & https://www.revistas.ufg.br/ci \\
\hline Em Questão & $1807-8893$ & http://seer.ufrgs.br/emquestao/ \\
\hline Informação \& Informação & $1981-8920$ & http://www.uel.br/revistas/uel/index.php/informacao \\
\hline Informação@Profissões & $2317-4390$ & http://www.uel.br/revistas/uel/index.php/infoprof \\
\hline Liinc em revista & $1808-3536$ & http://revista.ibict.br/liinc \\
\hline Logeion: filosofia da informação & 2358-7806 & http://revista.ibict.br/index.php/fiinf/index \\
\hline Perspectiva em $\mathrm{Cl}$ & $1413-9936$ & http://portaldeperiodicos.eci.ufmg.br/index.php/pci \\
\hline Ponto de Acesso & $1981-6766$ & https://portalseer.ufba.br/index.php/revistaici \\
\hline
\end{tabular}

Fonte: Dados da pesquisa.

A terceira etapa consistiu a coleta de dados altmétricos diretamente dos artigos publicados nos periódicos selecionados conforme os critérios de inclusão acima referidos (artigos de periódicos indexados na BRAPCI que contenham o DOI). O processo de coleta foi feito com base da ferramenta Bookmarklet for Researchers - Altmetric it! ${ }^{1}$ nos artigos publicados nos periódicos elegíveis, ou seja, naqueles em que o DOI tenha sido identificado e que, também, atribuem esse identificador aos artigos neles publicados.

A coleta de dados abarcou um período de seis anos (2012 - 2017), e nesses periódicos foram analisados todos os artigos publicados nesse espaço temporal. Vale salientar que, não foi possível coletar dados anteriores ao ano de 2014 no periódico Ponto de Acesso e dados anteriores ao ano de 2015 no Journal of Information Science: Research Trends, pois, em ambos periódicos, os artigos publicados anteriores a estes anos não possuem o DOI.

A opção pelo recorte inicial, 2012, deveu-se à constatação de que foi nesse ano em que o movimento altmetrics ganhou maior visibilidade no contexto internacional (COSTAS; ZAHEDI; WOUTERS, 2015) e, 2017, pela necessidade que se impõe de se trabalhar com dados

\footnotetext{
${ }^{1}$ Esta ferramenta se encontra disponível em <https://www.altmetric.com > e possui uma extensão no navegador Google Chrome para a coleta de dados em mídias sócias online (Facebook, Twitter e Google+), redes de compartilhamento (reddit) e em gestores de referências (Mendeley, Connoetea, Zotero, CiteULike) (GOUVEIA, 2013).
} 
atualizados, visto que as altmétricas identificam o impacto imediato das publicações científicas nos serviços de RSO.

A quarta etapa consistiu na análise de dados que teve como base a análise de conteúdo categorial de Bardin (1977), em que as categorias de análise foram definidas a priori, segundo a seguinte estrutura: i) aderência dos dados altmétricos na $\mathrm{Cl}$ brasileira, ii) mapeamento do tipo de dados na $\mathrm{Cl}$ brasileira, e iii) dados demográficos dos usuários das pesquisas da $\mathrm{Cl}$ do Brasil.

\section{Resultados e discussão}

Os resultados deste estudo são apresentados em três etapas, inter-relacionadas. A primeira contém informações sobre a aderência dos dados de altmetria nos periódicos da $\mathrm{Cl}$ indexados na $\mathrm{BRAPCl}$, estabelecendo a relação entre a visibilidade do periódico nas redes socais e o extrato no Qualis-Periódico. Na segunda, apresenta-se o mapeamento dos dados altmétricos identificados em cada periódico científico, com a finalidade de identificar quais indicadores as publicações da $\mathrm{Cl}$ apresentaram maior visibilidade. Por fim, na última seção apresenta-se os dados demográficos dos indivíduos que mencionaram ou compartilharam as publicações da BRAPCI nos serviços de RSO, bem como em ferramentas de gerenciamento de referência.

\subsection{Aderência dos dados de altmetria nos periódicos da Cl}

Considerando o número reduzido de periódicos brasileiros que possuem o DOI, é possível afirmar que ainda prevalecem algumas barreiras técnicas que impedem que se faça uma análise holística na visibilidade das publicações da $\mathrm{Cl}$ nos serviços de RSO e em outras plataformas digitais de compartilhamento e gerenciamento de informação.

Vale ressaltar que a dependência por identificadores tem sido um sido um dos grandes questionamentos que têm sido colocados relativamente à eficácia das altmétricas para avaliar com objetividade o impacto social das publicações científicas (HAUSTEIN, 2014; 2016; BORREGO, 2014).

A tabela 1 apresenta a síntese dos artigos analisados, exibindo o número total de artigos analisados em cada periódico da amostra, a soma do número de menções que estes artigos obtiveram nos serviços de RSO (Facebook, Twitter e blogs) e nas plataformas de gerenciamento de referências (Mendeley). 
Tabela 1 - Resultados de menções dos artigos por periódicos, classificados pela maior ocorrência de menções

\begin{tabular}{|c|c|c|c|}
\hline \multirow{2}{*}{ Nome do periódico } & \multirow{2}{*}{ ISSN } & \multicolumn{2}{|c|}{ Número de artigos } \\
\hline & & Analisados & Mencionados \\
\hline Informação \& Informação & $1981-8920$ & 225 & 25 \\
\hline Em Questão & $1807-8893$ & 121 & 12 \\
\hline Biblioteca Escolar em Revista & $2238-5894$ & 45 & 4 \\
\hline Informação@Profissões & $2317-4390$ & 68 & 1 \\
\hline Atoz: novas práticas de informação e conhecimento & 2237-826X & 60 & 1 \\
\hline Perspectiva em $\mathrm{Cl}$ & $1413-9936$ & 41 & 1 \\
\hline Liinc em revista & $1808-3536$ & 220 & 0 \\
\hline Ponto de Acesso & $1981-6766$ & 135 & 0 \\
\hline Brazilian Journal of Information Science: research trends & $1981-1640$ & 125 & 0 \\
\hline Ciência da Informação & 0100-1965 & 113 & 0 \\
\hline Comunicação \& Informação & $1415-5842$ & 110 & 0 \\
\hline Logeion: filosofia da informação & $2358-7806$ & 61 & 0 \\
\hline Total & - & 1324 & 44 \\
\hline
\end{tabular}

Fonte: Elaborado pelos autores.

Nos doze periódicos foram analisados todos os artigos publicados no período de 2012 a 2017, perfazendo um corpus constituído por um total de 1324 artigos analisados. Importa frisar que o quantitativo de artigos analisados por periódico não é uniforme por duas razões. Primeiro, porque o número de artigos publicados por edição varia de periódico para periódico. Isto é, as revistas que apresentam o número elevado de artigos analisados são as que têm uma média elevada de artigos por edição e que publicam várias edições por ano. Segundo, embora os periódicos tenham o $\mathrm{DOI}$, nem sempre os artigos neles publicados contém esse identificador. Portanto, várias publicações desses periódicos não fizerem parte do corpus da pesquisa por não possuir um identificador capaz de fornecer dados altmétricos.

Conforme a Tabela 1 , somente 5 (cinco) periódicos dispõem de publicações mencionadas nos serviços de RSO e em ferramentas de gerenciamento de referências, destacando-se a revista Informação \& Informação, que foi a que teve maior número de menções - o periódico com maior número de menções.

Nota-se, que os dois periódicos com maior quantidade de menções (Informação \& Informação e Em Questão) são parte integrante dos periódicos considerados de excelência da $\mathrm{Cl}$ brasileira segundo a avaliação do Qualis-Periódicos, pois estão classificados no extrato $A 2$, na área de Comunicação e Informação - a mesma que congrega os periódicos de $\mathrm{Cl}$.

Porém, não se pode estabelecer uma relação de causa-efeito entre a quantidade de menções e o extrato do periódico, pois a amostra contém outros periódicos classificados em 
extratos mais altos com pouca representatividade no total de menções. Em contraponto, o periódico Biblioteca Escolar em Revista não possui classificação no extrato Qualis-Periódico e está em terceiro lugar na lista dos periódicos com maior número de menções e compartilhamento de suas publicações.

O total de 44 (quarenta e quatro) menções ainda é considerado incipiente, trazendo à tona a necessidade de estudos aprofundados sobre as motivações que levam indivíduos a mencionar e/ou compartilhar determinadas publicações.

\subsection{Tipos de dados altmétricos presentes na BRAPCI}

Uma das constatações desta pesquisa revela a baixa inserção destas medidas de impacto social na $\mathrm{Cl}$ brasileira. Esta conclusão se fundamenta no fato de que no total de 1324 artigos analisados, somente 44 (quarenta e quatro) foram mencionados nas redes sociais online ou compartilhados em ferramentas de gerenciamento de referências. Ou seja, apenas 3.3\% (três por cento e três décimos) dos artigos publicados tiveram alguma interação nas RSO e em demais plataformas de interação social.

Os resultados também permitem a coleta de dados segregada dos indicadores para cada origem, contendo a quantidade de menções de publicações da $\mathrm{Cl}$ : no Mendeley, no Twitter, no Facebook e nos blogs (Tabela 2). Estes dados ressaltam a atual preferência de compartilhar informações científicas no Mendeley, que é uma ferramenta online de gerenciamento de referências que, nos últimos anos, tem sido muito utilizada no contexto acadêmico.

Tabela 2 - Distribuição geral das informações altmétricas por cada plataforma online

\begin{tabular}{|c|c|}
\hline Fontes de dados altmétricos & Quantidade de menções \\
\hline Mendeley & 89 \\
\hline Twitter & 25 \\
\hline Facebook & 24 \\
\hline Blogs & 4 \\
\hline Total & 152 \\
\hline
\end{tabular}

Fonte: Elaborado pelos autores.

Esta constatação é similar à de outros estudos realizados em bases de dados internacionais, tais como Web of Science (WoS) e Scopus, que coincidem no fato de que o gestor de referências bibliográficas Mendeley é a fonte de dados altmétricos que inclui maior volume de produção científica (BORREGO, 2014). Em pesquisa semelhante, Zahedi, Costas e Wouters (2014) concluíram que cerca de 63\% (sessenta e três) dos artigos indexados na WoS 
entre 2005 e 2009 estão incluídos em alguma conta de Mendeley, embora com diferenças disciplinares.

No que tange aos serviços de RSO, verifica-se que o Twitter é a plataforma em que a produção científica da $\mathrm{Cl}$ do Brasil capta maior atenção, por meio de compartilhamentos e menções de artigos nas postagens. Araújo (2015) obteve a mesma constatação em pesquisa na qual pretendia analisar as métricas alternativas de um número limitado de artigos publicados em periódicos do extrato $\mathrm{A} 1$ da $\mathrm{Cl}$ brasileira.

Esta conclusão reflete-se em outros estudos no contexto internacional (ADIE; ROE, 2013; COSTAS; ZAHEDI; WOUTERS, 2014) realizados em diversos periódicos disciplinares e interdisciplinares, que constataram que o Twitter, seguido pelo Facebook, tem sido os serviços de RSO que mais mencionam publicações científicas. Este fenômeno está relacionado ao fato de estas RSO serem as que possuem maior quantidade de usuários, em escala global.

No que diz respeito aos periódicos científicos, destaca-se o periódico "Informação \& Informação" que concentra a maior parte de dados altmétricos coletados por este estudo e, inclusive, foi nesse periódico em que foi encontrado o artigo com o maior número de menções nas mídias sociais online e nas ferramentas de gerenciamento de referências - Mendeley.

A Tabela 3 exibe os elementos relativos à evolução temporal dos dados altmétricos em cada periódico, no intervalo que compreende os anos 2012 e 2017. Verifica-se a ausência de menções e compartilhamentos de artigos em plataformas online no período de 2012 a 2014, pois seguindo os argumentos de vários autores (COSTAS; ZAHEDI; WOUTERS, 2014; HAUSTEIN, 2016; ALPERIN, 2013), nesta fase, estas métricas ainda careciam de uma base teórica sólida, aliado ao fato de as mesmas serem completamente dependentes de identificadores (DOI e outros) nem sempre acessíveis para os periódicos fora do contexto que representa a chamada ciência mainstream. A tabela em alusão mostra que os primeiros dados de menções e compartilhamentos de artigos nas plataformas de redes sociais online ocorreram, de forma "tímida", em 2015 e, com maior vigor, a partir de 2016.

Tabela 3 - Distribuição das altmétricas ao longo do tempo

\begin{tabular}{lrrrrrrrr}
\hline Nome do periódico & ISSN & $\mathbf{2 0 1 2}$ & $\mathbf{2 0 1 3}$ & $\mathbf{2 0 1 4}$ & $\mathbf{2 0 1 5}$ & $\mathbf{2 0 1 6}$ & $\mathbf{2 0 1 7}$ & Total \\
\hline Informação \& Informação & $1981-8920$ & 0 & 0 & 0 & 14 & 94 & 6 & 114 \\
Em Questão & $1807-8893$ & 0 & 0 & 0 & 0 & 8 & 14 & 22 \\
A to z: novas práticas de informação e & $2237-826 X$ & 0 & 0 & 0 & 0 & 8 & 0 & 8 \\
conhecimento & $2238-5894$ & 0 & 0 & 0 & 0 & 2 & 2 & 4 \\
Biblioteca Escolar em Revista & $2317-4390$ & 0 & 0 & 0 & 3 & 0 & 0 & 3 \\
Informação@Profissões & $1413-9936$ & 0 & 0 & 0 & 0 & 0 & 1 & 1 \\
Perspectiva em CI & & & & & & &
\end{tabular}




\begin{tabular}{|c|c|c|c|c|c|c|c|c|}
\hline Nome do periódico & ISSN & 2012 & 2013 & 2014 & 2015 & 2016 & 2017 & Total \\
\hline $\begin{array}{l}\text { Brazilian Journal of Information } \\
\text { Science: research trends }\end{array}$ & $1981-1640$ & 0 & 0 & 0 & 0 & 0 & 0 & 0 \\
\hline Ciência da Informação & 0100-1965 & 0 & 0 & 0 & 0 & 0 & 0 & 0 \\
\hline Comunicação \& Informação & $1415-5842$ & 0 & 0 & 0 & 0 & 0 & 0 & 0 \\
\hline Liinc em revista & $1808-3536$ & 0 & 0 & 0 & 0 & 0 & 0 & 0 \\
\hline Logeion: filosofia da informação & $2358-7806$ & 0 & 0 & 0 & 0 & 0 & 0 & 0 \\
\hline Ponto de Acesso & $1981-6766$ & 0 & 0 & 0 & 0 & 0 & 0 & 0 \\
\hline Total & - & 0 & 0 & 0 & 17 & 112 & 23 & 152 \\
\hline
\end{tabular}

Fonte: Dados da pesquisa

Estes aspectos demonstram a inserção recente destas métricas no contexto brasileiro, principalmente nos anos entre 2015 e 2017 e, concomitante à visão de Araújo (2014), está associada ao aumento do número de pesquisadores cada vez mais envolvidos com serviços de RSO, conscientes da visibilidade que estas plataformas atribuem às pesquisas e aos respectivos autores.

No entanto, é necessário um aprofundamento teórico acerca destes indicadores para a ampliação do uso e aceitação pelas comunidades científicas e, sobretudo no sentido de combiná-los com os indicadores tradicionais, baseados em citações.

\subsection{Dados demográficos das menções aos periódicos analisados}

Um dos aspectos relevantes da utilização uso de métricas alternativas é a possibilidade de acesso a dados de mapeamento demográfico sobre os indivíduos que estão utilizando e/ou acessando as publicações científicas. 
Figura 1 - Acesso aos dados demográficos

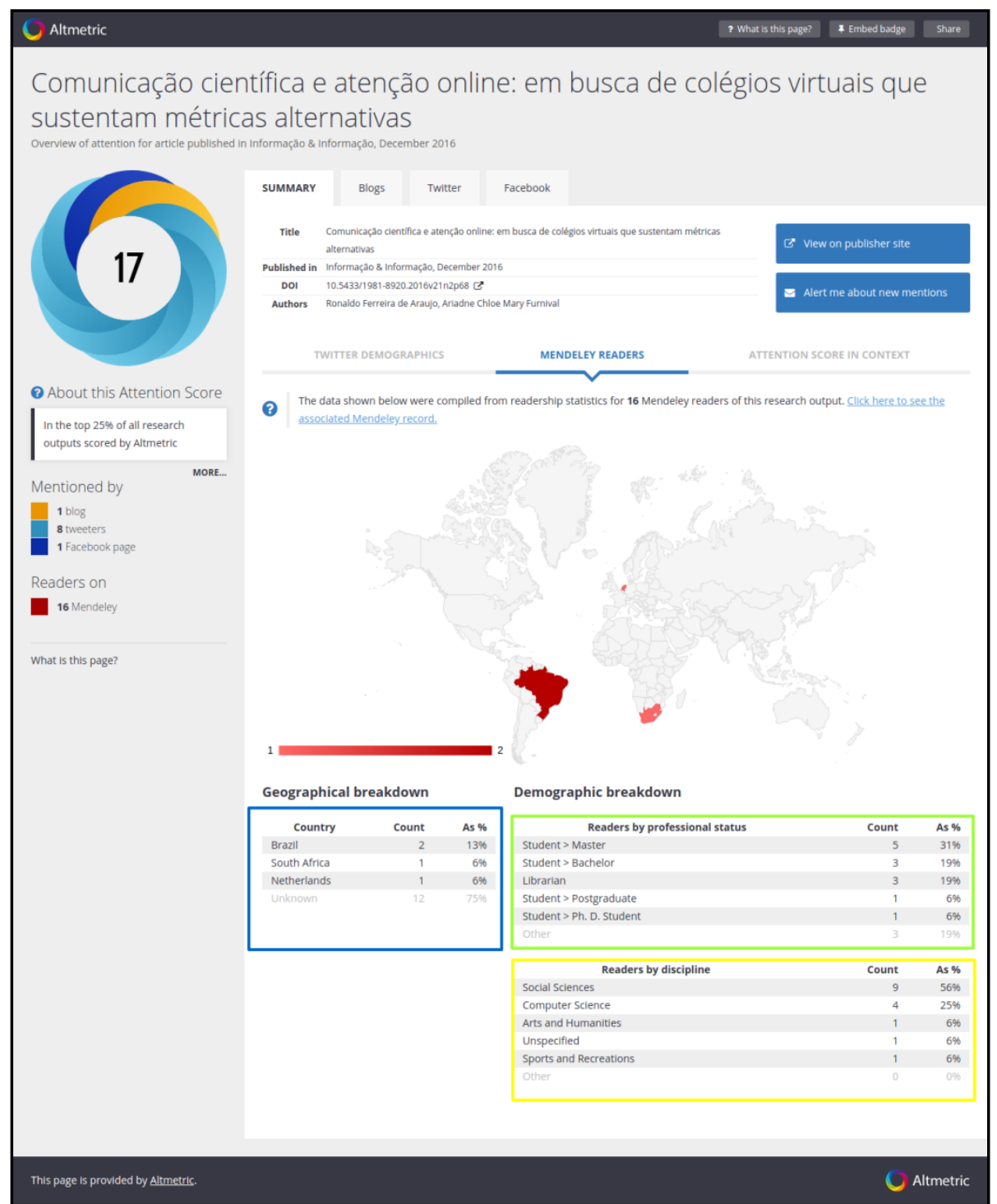

Fonte: Recorte de Altmetrics (2018a), por autores.

A ferramenta disponibiliza dados demográficos (Figura 2) segmentados por país de origem do acesso ao artigo (retângulo azul), uma categorização para segmentar perfis de usuário (retângulo verde), incluindo as respectivas áreas do conhecimento a que se encontram vinculados (retângulo amarelo). É necessário ressaltar que as únicas fontes utilizadas pela Altmetric.com que fornecem dados demográficos são o Twitter e o Mendeley e que mesmo assim há diferenças nas informações entre eles quanto a caracterização do perfil de usuários.

Entretanto, a coleta de dados demográficos ainda apresenta desafios, na medida em que os dados disponíveis para a coleta são apenas disponibilizados pelos usuários, seja no ato do cadastro ou em outras circunstâncias (MCCORMICK et al., 2015). Como corolário dessa limitante, dos artigos analisados nesta pesquisa verificou-se que aproximadamente $80 \%$ (oitenta por cento) dos gerenciamentos no Mendeley e 30\% (trinta por cento) das menções no 
Twitter, a localização dos usuários aparece como desconhecida, pois podem ser anonimidades referentes ao uso de ferramentas de ofuscamento de dados pessoais, como os proxies.

Além disso, os elementos demográficos disponibilizados por esta ferramenta não fornecem informações sobre gênero e faixa etária que, segundo McCormick et al. (2015), constituem elementos essenciais da coleta de dados em pesquisas relacionadas a estudos das ciências sociais.

\begin{tabular}{lr}
$\begin{array}{l}\text { Tabela 4 - Áreas do conhecimento dos usuários que } \\
\text { salvaram as publicações no Mendeley }\end{array}$ \\
\hline Área do Conhecimento & $\begin{array}{r}\text { Quantidade de } \\
\text { Menções }\end{array}$ \\
\hline Ciências Sociais & 45 \\
Ciências da Computação & 25 \\
Artes e Humanidades & 10 \\
Auditores, Gerentes e Empresários & 3 \\
Ciências Agrárias e Biológicas & 3 \\
Desporto e recreação & 2 \\
Economia, Econometria e Finanças & 1 \\
Matemática & 1 \\
Física e Astronomia & 1 \\
Ciências da Decisão & 1 \\
Engenharia & 1 \\
Ciências Ambientais & 1 \\
\hline Total & 94
\end{tabular}

Fonte: Elaborado pelos autores.

A Tabela 4 exibe uma compilação contendo dados sobre as áreas do conhecimento dos usuários que mencionaram as publicações analisadas. Importa considerar que estes dados são apenas disponibilizados pelo Mendeley, não sendo possível a sua obtenção nos serviços de RSO, tais como Facebook e Twitter.

Embora os periódicos sejam majoritariamente acessados por indivíduos das Ciências Sociais, observa-se, também, o caráter trans e interdisciplinar da $\mathrm{Cl}$, sobretudo com as Ciências da Computação, bem como com as diversas disciplinas das Ciências Humanas e de outras áreas correlatas.

A interdisciplinaridade da $\mathrm{Cl}$ diz respeito à complexidade do seu objeto de estudo que é, também, abordado por outras disciplinas com enfoques particulares. Esta intercessão da $\mathrm{Cl}$ com outras disciplinas faz com que partilhem, dentre outros aspectos, abordagens teóricas e metodológicas. Conforme Saracevic (1996), Cl é interdisciplinar por natureza, porque os profissionais responsáveis pela fundação deste campo eram provenientes de outras áreas, com 
destaque para a Biblioteconomia, a Educação, a Psicologia, a Computação. Na visão de Santos (1986) e Morin (2005), o diálogo entre as áreas do conhecimento é uma das exigências da ciência pós-moderna.

Tabela 5 - Atuação profissional dos usuários que mencionaram as publicações

\begin{tabular}{lr}
\hline Atuação profissional & $\begin{array}{r}\text { Quantidade de } \\
\text { Menções }\end{array}$ \\
\hline Estudante de Mestrado & 23 \\
Bibliotecário & 20 \\
Estudante de Doutorado & 14 \\
Membro do público & 14 \\
Estudante de Pós-Graduação & 12 \\
Estudante de Graduação & 11 \\
Professor & 9 \\
Comunicadores de Ciência (Jornalistas, Blogueiros e Editores) & 7 \\
Cientista & 2 \\
Pesquisador & 2 \\
Outro & 2 \\
\hline Total & 116 \\
\hline
\end{tabular}

Fonte: Elaborado pelos autores.

A Tabela 5 exibe uma compilação contendo dados sobre a atuação profissional dos usuários que mencionaram as publicações analisadas. O perfil profissional dos usuários das RSO que mais mencionaram as publicações dos periódicos brasileiros indexados na BRAPCl é constituído por pessoas envolvidas no meio acadêmico, em particular, bibliotecários, estudantes de graduação e de pós-graduação e professores. Destaque para o fato de a categoria "Membro do público" aparecer em realce, o que justifica a natureza das altmétricas, cuja motivação de seus proponentes e defensores é demonstrar o impacto das publicações científicas dentro e fora do contexto acadêmico.

Sintetizando, as métricas alternativas, embora sejam pensadas como indicadores para impulsionar a visibilidade da produção científica dos países em desenvolvimento (ALPERIN, 2013), esta investigação evidenciou que, em linhas gerais, elas ainda em fase embrionária para os periódicos brasileiros da $\mathrm{Cl}$. Este cenário pode estar associado às barreiras anteriormente mencionadas para a obtenção do DOI, baixa quantidade de menções de publicações brasileiras nos serviços de RSO, bem como a pouca popularidade que as altmetrias têm dentro da academia.

Por outro lado, existe a inevitabilidade de seu aprofundamento teórico e metodológico para que pesquisadores, editores e agências de fomento da pesquisa tenham 
consciência sobre a importância dos serviços de RSO como espaços onde interlocutores - não só originários da academia - possam compartilhar e debater o conhecimento produzido, além dos canais de comunicação tradicionais.

Dessa forma, postulamos que, enquanto estas limitações prevalecerem, estes indicadores terão uma contribuição pequena ao objetivo inicial de democratização da recompensa acadêmica e da promoção da visibilidade da ciência periférica, podendo contribuir para sedimentação do cenário da exclusão cognitiva já patente no sistema tradicional de avaliação da atividade científica.

\section{Considerações finais}

Com maior penetrabilidade das TIC nas ações e atividades humanas, diversos aspectos da comunicação científica passaram por transformações. Entre estas transformações este texto destaca a inserção dos periódicos nos ambientes web e as métricas alternativas altmetrias - como novo instrumento para auxiliar a avaliação do impacto das publicações científicas dentro e fora do âmbito acadêmico.

Neste estudo, a primeira constatação se relaciona à prevalência de algumas barreiras técnicas, pois a coleta dos dados altmétricos depende, exclusivamente, da existência de um identificador padrão, em particular, a adoção do DOI. Aproximadamente 80\% (oitenta por cento) dos periódicos científicos indexados na BRAPCl ainda não possui esse identificador, fator limitante para a identificação de menções as publicações nos serviços de RSO. Dos periódicos analisados, menos de metade possuem dados altmétricos, o que sugere a pouca inserção de conteúdos das publicações fora do âmbito acadêmico.

Os dados altmétricos analisados apresentaram maior concentração nos serviços de RSO Twitter e Facebook. Vale ressaltar que o uso de serviços de RSO para divulgação de conteúdos científicos não é um cenário exclusivo do Brasil, pois estudos de outros países também apresentaram resultados semelhantes. Por outro lado, esse cenário pode ser associado ao fato destes serviços de RSO serem os de maior popularidade na maioria dos países.

Dos artigos analisados, apenas 3\% (três por cento) apresentam, pelo menos, um dado altmétrico. Isso sugere que apesar do movimento das métricas alternativas se encontrar em fase de consolidação, no contexto específico do Brasil estas ainda estão em fase embrionária, o que retarda a possibilidade das mesmas se tornarem alternativas válidas aos processos hegemônicos de avaliação da atividade científica

Perante estas circunstâncias, há necessidade de se conscientizar a comunidade acadêmica, em particular editores e agências de fomento sobre a importância destas métricas 
alternativas como novo subsídio para a avaliação do impacto de produtos científicos dentro e fora do contexto acadêmico.

\section{Referências}

ADIE, E; ROE, W. Altmetric: enriching scholarly content with article-level discussion and metrics. Learned publishing, v. 26, n. 1, p. 11-17, Jan. 2013. Disponível em: <http://onlinelibrary.wiley.com/doi/10.1087/20130103/abstract>. Acesso em: 11 nov. 2017.

ALPERIN, J. P. Ask not what altmetrics can do for you, but what altmetrics can do for developing countries. Bulletin of the Association for Information Science and Technology, v. 39, n. 4, p. 18-21, 2013. Disponível em: <http://onlinelibrary.wiley.com/doi/10.1002/bult.2013.1720390407/epdf >. Acesso em: 02 out. 2017.

ALTMETRIC. Digital Science \& Research Ltd. Bookmarklet for Researchers - Altmetric it!. Altmetric - Comunicação científica e atenção online: em busca de colégios virtuais que sustentam métricas alternativas [recurso de dados]. Estados Unidos da América, 2018a. Disponível em: <https://www.altmetric.com/details/14895345?src=bookmarklet $>$. Acesso em: 23 maio 2018.

ALTMETRIC. Digital Science \& Research Ltd. Bookmarklet for Researchers - Altmetric it!. Estados Unidos da América, 2018b. Disponível em: $<$ https://www.altmetric.com/products/freetools/bookmarklet/>. Acesso em: 23 maio. 2018.

ARAÚJO, R. F. Cientometria 2.0, visibilidade e citação: uma incursão altmétrica em artigos de periódicos da Cl. Encontro Brasileiro de Bibliometria e Cientometria, v. 4, p. 1-8, 2014. Disponível em:

<http://www.brapci.inf.br/index.php/article/view/0000014387/85d840bd07c105f25556f0b6e9 18d13e />. Acesso em: 11 nov. 2017.

ARAÚJO, R. F. Mídias sociais e comunicação científica: análise altmétrica em artigos de periódicos da ciência da informação. Em Questão, v. 21, p. 96, 2015. Disponível em: <https://doi.org/10.19132/1808-5245211.96-109>.

BORREGO, A. Altmétricas para la evaluación de la investigación y el análisis de necesidades de información. El Professional de Información, Julio-agosto, V. 23, n. 4, p. 352-257, 2014.

BORNMANN, L. Do altmetrics point to the broader impact of research? An overview of benefits and disadvantages of altmetrics. Journal of Informetrics, v. 8, p. 895-903, 2014. Disponível em: <https://www.sciencedirect.com/science/article/pii/S1751157714000868>. Acesso em: 17 nov. 2017.

COSTAS, R; ZAHEDI, Z; WOUTERS, P. Do "altmetrics" correlate with citations? Extensive comparison of altmetric indicators with citations from a multidisciplinary perspective. Journal of the Association for Information Science and Technology, v. 66, n. 10, p. 2003-2019, 2015. Disponível em <https://onlinelibrary.wiley.com/doi/abs/10.1002/asi.23309>, acesso 16 Maio 2018.

GOUVEIA, F. C. Altmetria: métricas de produção científica para além das citações. Liinc em Revista, Rio de Janeiro, v. 9, n. 1, p. 214-227, maio 2013. Disponível em: <http://www.ibict.br/liinc>. Acesso em: 03 out. 2017.

HAUSTEIN, S. Grand challenges in altmetrics: heterogeneity, data quality and dependencies. Scientometrics, v. 108, n. 1, p. 413-423, Mar. 2016. Disponível em: <https://link.springer.com/article/10.1007/s11192-016-1910-9>. Acesso 03 out. 2017. 
HAUSTEIN, S. et. al. Coverage and adoption of altmetrics sources in the bibliometric community. Scientometrics, v. 101, n. 2, p. 1145-1163. Disponível em <https://link.springer.com/article/10.1007/s11192-013-1221-3>, acesso 16 Maio 2018.

KURAMOTO, H. Acesso livre: um caso de soberania nacional? In: TOUTAIN, L. M. B. B. (Org.). Para entender a Ciência da Informação. Salvador: EDUFBA, 2007. p. 145-161.

MACIAS-CHAPULA, C. A. O papel da informetria e da cienciometria e sua perspectiva nacional e internacional. Ciência da Informação, Brasília, v. 27, n. 2, p. 134-140, maio/ago. 1998. Disponível em: <http://www.scielo.br/pdf/ci/v27n2/macias.pdf>. Acesso 13 jul. 2017.

McCORMICK, T. H. et al. Using twitter for demographic and social science research: tools for data collection and processing. Sociological methods \& research, v. 46, n. 3, p. 390-421, August 2015. Disponível em: <http://journals.sagepub.com/doi/abs/10.1177/0049124115605339>. Acesso 22 set. 2017.

McFEDRIES, P. Measuring the impact of altmetrics. IEEE Spectrum, v. 49, n. 8, p. 28, August, 2012. Disponível em: $\langle$ http://ieeexplore.ieee.org/stamp/stamp.jsp?arnumber=6247557> Acesso em: 13 out. 2017.

MEADOWS, A. J. A comunicação científica. Brasília: Briquet de Lemos, 1999. 268 p.

MORIN, Edgar. Ciência com Consciência. 8 ed. -Rio de Janeiro: Bertrand, 2005.

PRIEM, J.; HEMMINGER, B.M. Scientometrics 2.0: toward new metrics of scholarly impact on the social web. First monday, v. 15, n. 7, Nov. 2010. Disponível em: <http://firstmonday.org/article/view/2874/2570>. Acesso em: 21 set. 2017.

PRIEM, J. et al. Altmetrics: a manifesto, out. 2010. Disponível em: <http://altmetrics.org/manifesto>. Acesso, 20 out. 2017.

ROBINSON-GARCÍA, N. et al. New data, new possibilities: exploring the insides of Altmetric.com. El profesional de la información, v. 23, n. 4, p. 359-366, jul. 2014. Disponível em: <https://arxiv.org/abs/1408.0135>. Acesso 03 out. 2017.

RODRIGUES, F. A. Coleta de dados em redes sociais: privacidade de dados pessoais no acesso via Application Programming Interface. Tese. Marília, Brasil: Universidade Estadual Paulista, 3 mar. 2017.

RODRIGUES, F. A.; SANT'ANA, R. C. G. Use of Taxonomy of Privacy to Identify Activities Found in Social Network's Terms of Use. Knowledge Organization, v. 43, n. 4, p. 285-295, 2016.

SANTOS, B de S. Um discurso sobre as ciências na transição para uma ciência pós-moderna. Estudos Avançados. São Paulo, v. 2,n. 2, Aug. 1988.

SMALL, H. On the shoulders of Robert Merton: towards a normative theory of citation. Scientometrics, v. 60, n.1, p. 71-79, 2004.

SPINAK. E. Indicadores Cienciométricos. Ciência da Informação, Brasília, v. 27, n. 2, p. 141-148, 1998.

VANTI, N.; SANZ-CASADO, E. Altmetria: a métrica social a serviço de uma ciência mais democrática. TransInformação, Campinas, v. 28, n. 3, p. 349-358, set./dez., 2016. Disponível em: < http://www.scielo.br/scielo.php?pid=S0103-

37862016000300349\&script=sci_abstract\&tlng=pt> Acesso em: 3 out. 2017. 